\title{
BMJ Open Applied investigation of person-specific and context-specific factors on postoperative recovery and clinical outcomes of patients undergoing gastrointestinal cancer surgery: multicentre European study
}

To cite: Markar SR, Mavroveli S, Petrides KV, et al. Applied investigation of person-specific and contextspecific factors on postoperative recovery and clinical outcomes of patients undergoing gastrointestinal cancer surgery: multicentre European study. BMJ Open 2016:6:e012236.

doi:10.1136/bmjopen-2016012236

- Prepublication history and additional material is available. To view please visit the journal (http://dx.doi.org/ 10.1136/bmjopen-2016012236).

Received 10 April 2016 Revised 21 June 2016 Accepted 25 July 2016

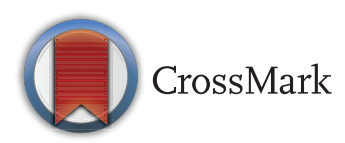

For numbered affiliations see end of article.

Correspondence to Professor George B Hanna; g.hanna@imperial.ac.uk

\section{ABSTRACT}

Introduction: Cancer treatments have greatly advanced over the past two decades causing survival improvements and reduced complications from cancer surgery. However, the cancer diagnosis and the effects of treatment modalities pose a major risk to patients' psychological well-being. Given current interest and emerging evidence about the importance of psychological and social factors on cancer survival and coping with cancer treatments, this study will build and expand research in order to identify key modifiable psychosocial variables that contribute to better physical and mental health following gastrointestinal cancer (GIC) surgery.

Objectives: To elucidate the incidence of postoperative psychiatric morbidity within 6 months following GIC surgery. To identify key measurable modifiable preoperative psychological factors that can significantly affect postoperative psychiatric morbidity in patients undergoing surgery for GIC. To clarify the changes seen in a patient's psychological well-being during their treatment pathway for GIC.

Methods and analysis: This multicentre study has an observational longitudinal study design. In total, 1000 patients will be screened with a multicomponent psychological questionnaire at four different time points: at diagnosis, preoperatively, 1 and 6 months after surgery. Data from this questionnaire will be linked to postoperative complications including psychiatric morbidity, length of hospital stay and recovery to normal activity.

Ethics and dissemination: NHS Health Research Authority approval was gained on (REC reference 15. LO/1847) for the completion of this study. Multiple platforms will be used for the dissemination of the research data, including international clinical and patient group presentations and publication of research outputs in a high impact clinical journal.

\section{INTRODUCTION}

Cancer diagnosis and treatment have a profound effect on patients' physical and psychological well-being. ${ }^{1-4}$ Patients can experience elevated stress and anxiety levels, depressive symptomatology, psychosomatic symptoms and, subsequently, may adopt maladaptive coping strategies, ${ }^{5-7}$ leading to poorer cancer outcomes and survival rates. ${ }^{8-11}$ Depression has been linked to increased mortality risk in patients with lung cancer, ${ }^{11}$ while convergent evidence suggests its prevalence among this group of patients can be as high as $40 \% .{ }^{12}$ In a national UK study we showed that the rate of psychiatric morbidity was significantly higher in patients with GIC even after controlling for preoperative psychiatric morbidity. Furthermore, when adjusted for covariates preoperative psychiatric diagnosis made a unique and significant contribution to longterm mortality rates following cancer surgery. Similar effects were observed for postoperative psychiatric morbidity. ${ }^{1}$ These findings were paralleled in a Swedish cohort study, which showed new-onset psychiatric morbidity after oesophagectomy was associated with increased mortality. ${ }^{13}$

The mechanisms by which psychological variables, such as anxiety and depression, impact patient cancer outcomes and, especially, survival are still unclear, ${ }^{12}$ and underinvestigated. For the relationship between depression and poor outcomes a number of speculative explanations have been proposed, such as psychobiological effects via endocrine or immunological pathways, amplified 
depression severity due to symptom seriousness and lack of compliance with treatment protocol as an outcome of depression.

In a comprehensive review study, a number of psychological variables, such as social support, marriage, emotion constraint as well as depression, have been linked to breast cancer survival. ${ }^{14}$ The impact of personality on cancer onset and survival has also attracted scientific attention. However, methodological limitations (eg, the use of short questionnaires due to time constraints) and failure to control for confounding variables, ${ }^{12}$ have hindered the discovery of direct causal effects between psychosocial variables and cancer onset and survival. Current evidence is predominantly based on cross-sectional studies, small sample sizes and extensive variations in psychological parameters and outcome metrics across studies. ${ }^{14}{ }^{15}$ Furthermore, the hypothesised causal associations remain unclear and warrant further investigation. ${ }^{12} 1617$

No study thus far has investigated the patient cancer trajectory and how different cancer treatment modalities may have differential impact on patient onset of psychiatric morbidity, although some relevant data from diagnostic pathways do exist. ${ }^{18}{ }^{19}$ Furthermore, the potential link between psychosocial factors and cancer gave rise to the development of interventions in order to alleviate psychological and physical distress and improve survival. ${ }^{20-23}$ However, detailed and reliable patient profiling prior to the development of tailored targeted interventions has been inexplicably overlooked.

Given the current interest and trends in the literature regarding the importance of person-specific and context-specific variables on cancer survival, our work will focus on investigating and identifying a wide range of modifiable psychosocial factors that may contribute to improved psychological and physical outcomes for patients during their cancer pathway. This comprehensive investigation aims to develop a patient profiling tool that can be used for the early identification of patients at risk of psychiatric morbidity. The tool will allow for the future development of tailored, targeted interventions before and following cancer treatment in order to reduce psychiatric morbidity and adverse cancer outcomes including mortality.

The importance of this study lies in the inclusion of an under-researched population (ie, patients with GIC), comprising patients who are likely to suffer life-altering effects as a result of their cancer treatment. Patients undergoing GIC surgery represent a unique patient group, as surgery fundamentally impacts several aspects of normal daily life including eating, bowel function, pain and levels of fatigue, with often prolonged effects on quality of life. ${ }^{24} 25$ The problem is heightened by the greater incidence of postoperative complications seen in GIC surgery compared to other studied types of surgery. ${ }^{24}{ }^{25}$ Also, to the best of our knowledge, this is the first prospective study to examine a number of potentially modifiable factors that clinicians and allied health professionals can use within the context of a cancer clinic in order to identify and profile their patients with the aim of providing targeted support. For these reasons, this project shares important clinical and public health implications.

\section{OBJECTIVES}

1. To elucidate the incidence of postoperative psychiatric morbidity within 6 months following GIC surgery.

2. To identify key measurable modifiable preoperative psychological factors that can significantly affect postoperative psychiatric morbidity in patients undergoing surgery for GIC.

3. To clarify the changes seen in a patient's psychological well-being during their treatment pathway for GIC.

\section{METHODS AND ANALYSIS \\ Study design}

This is a longitudinal study that will use a multifactorial psychological questionnaire (see online supplementary appendix A) to be administered at different time points in the patient treatment pathway (figure 1). Key factors from this questionnaire will be identified through correlation with outcomes of postoperative recovery in particular psychiatric morbidity.

\section{Patient pathway and recruitment}

Participants will be approached for recruitment after their consultation at a cancer or surgical clinic. The questionnaire will be completed individually. Testing sessions conducted by a research psychologist are expected to last $\sim 25 \mathrm{~min}$. Patients' emotional perceptions ('trait emotional intelligence'), coping, emotion regulation, anxiety and depression will be tested via the questionnaire. Information regarding participants' recovery outcomes and survival will be obtained from the medical records including postoperative complications and length of hospital stay. Patients will be screened typically four or five times along their patient pathway (figure 1); prior to any treatment, following neoadjuvant chemotherapy or chemoradiotherapy (if received), 1, 6 and 12 months following primary surgical resection of the GIC. Some patients with colorectal cancer may receive a defunctioning ileostomy at the time of GIC resection; however, this is most commonly reversed within 12 months of surgery and thus psychological screening will be performed in the presence and absence of a stoma.

Patients will be recruited from centres across Europe.

\section{Measures}

Archival data

Demographic information: Data on patient gender, age, body mass index, ethnic background, socioeconomic status (Carstairs index), smoking status, medical 
Figure 1 Patient pathway and timing of psychological screening test.

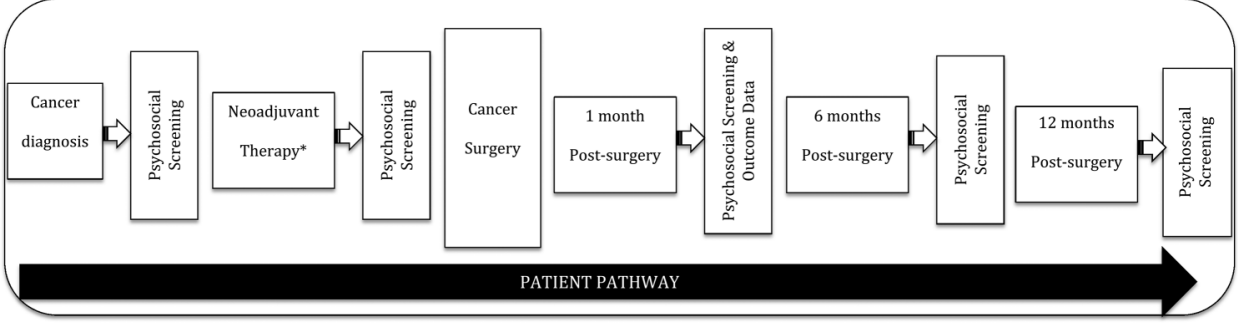

*A proportion of patients may progress directly to surgery and not receive neoadjuvant therapy; these patients will be sampled immediately before surgery and at 1 month, 6 months and 12 months after surgery comorbidities, GIC type (oesophageal, gastric or colorectal) and previous psychiatric diagnosis will be collected through review of medical records.

Tumour and treatment information: Information on stage, tumour location, type of surgery (including stoma formation) and neoadjuvant therapy used will be collected by reviewing medical records.

\section{Multifactorial psychological questionnaire Development of questionnaire}

All factors chosen for inclusion were selected as they have previously been shown to relate to psychological and/or physical well-being and these are also potentially modifiable psychological variables. Following an initial literature review, a consensus process and discussion was used to gain agreement from all authors and collaborators regarding the constructs included within the questionnaire. The questionnaire was then piloted in five patients, with feedback received and further modification of the questionnaire performed to generate the final questionnaire for use in the study (see online supplementary appendix A).

\section{Psychological factors included in questionnaire}

Trait Emotional Intelligence: The Trait Emotional Intelligence Questionnaire-Short Form (TEIQue-SF) ${ }^{26}$ comprises 30 items responded to a seven-point Likert scale. The instrument provides scores on well-being, selfcontrol, emotionality, sociability and global trait emotional intelligence.

Beck Depression Inventory (BDI-II). ${ }^{27}$ The BDI-II comprises 21 items assessing the severity of depression. Each item presents a list of four statements about a particular symptom of depression, arranged in increasing severity. A total score is calculated, with $0-13$ indicating minimal depression, 14-19 indicating mild depression, 20-28 indicating moderate depression and 29-63 indicating severe depression. The BDI has been used to assess depression for over 40 years, ${ }^{28}$ and has strong evidence of reliability and validity.

BRIEF-COPE. ${ }^{29}$ This is a 28-item questionnaire designed to assess 14 conceptually distinct methods of coping (active coping, self-distraction, denial, substance use, use of emotional support, use of instrumental support, venting, positive reframing planning, humour, acceptance, religion and self-blame). It is an abbreviated version of the COPE questionnaire and it has good psychometric properties.

State-Trait Anxiety Inventory (STAI)-Short Form. ${ }^{30}$ The STAI assesses an individual's conscious awareness at two extremes of anxiety affect, labelled state anxiety (A-state), and trait anxiety (A-trait), respectively. A-Trait can be defined as a relatively enduring disposition to feel stress, worry and discomfort and this is what we are measuring for the purposes of this study (20 items). The questionnaire has robust psychometric properties, and has been widely used across different populations. ${ }^{31}$

Expectancy-Value Questionnaire. This brief measure assesses health-related self-efficacy as well as healthrelated values. It is based on belief-importance theory, ${ }^{32}$ which has shown exceptionally strong predictive utility achieved through brief, unobtrusive and easily administrable instruments.

\section{Outcome data}

The primary outcome for this study is postoperative psychiatric morbidity within 30 days of surgery. Secondary outcomes for this study include, completion of intended neoadjuvant therapy, admission to hospital during neoadjuvant therapy, length of hospital stay following surgery, postoperative complications and recovery to normal activity in the community (SF-36) (assessed at the same time points as psychological questionnaire figure 1). SF-36 was chosen to permit comparison between different GICs. Standardised definition of complications will be used for the study to allow meaningful comparison of complications including psychiatric morbidity. ${ }^{33}$

\section{SYMPTOMATIC QUALITY OF LIFE QUESTIONNAIRE}

At the same time points as the psychological screening, patients will be asked to complete symptomatic quality of life questionnaires related to their primary diagnosis. For patients with colorectal cancer this will be the EORTC QLQ-CR29, ${ }^{34}$ for patients with oesophageal cancer this will be EORTC QLQ-OES24, ${ }^{35}$ and for patients with gastric cancer this will be EORTC QLQ-STO 22. ${ }^{36}$ 


\section{INCLUSION CRITERIA}

Patients undergoing intended curative surgery for the management of GIC.

\section{EXCLUSION CRITERIA}

1. Patients with metastatic GIC.

2. Patients undergoing non-curative surgery or no surgical intervention for GIC.

3. Patients undergoing surgery for a benign condition of the gastrointestinal tract.

\section{Sample size}

Based on the number of predictors $(\mathrm{k})$ in the logistic regression model and predicted prevalence rate of postoperative psychiatric morbidity $(\mathrm{p})$. Then:

$$
\text { Sample size }(\mathrm{n})=10 \times \mathrm{k} / \mathrm{p}
$$

With 9 covariates and a predicted prevalence rate of postoperative psychiatric morbidity of $12 \%$, the sample size required will be 750 patients.

We anticipate running patient recruitment for 10 months with a further 6 months follow-up. From the national audit figures for colorectal and oesophagogastric cancer, on average each participating European centre performs 18 GIC surgical resections per month; therefore, over the 10-month recruitment period 180 patients per centre will be screened. Eight centres will participate in the study giving an expected recruitment of 1440 patients; the expected dropout rate during the follow-up period is 25\%. Taking attrition into consideration the expected actual recruitment for the study will be 1080 patients.

\section{Statistical methodology}

Descriptive and exploratory analysis of statistics will be conducted to check the suitability of the data for inferential statistics. Scoring from the questionnaire will be analysed at different time points to observe changes in patients' psychological well-being during the course of their cancer treatment using linear mixed-effect models. Comparison across changes observed in each psychological area will be examined, in association with each time-point in the patient-treatment pathway using linear mixed-effect models.

Comparison of preoperative psychological factors from the questionnaire between patients with and without postoperative psychiatric morbidity will also be performed. Multivariable regression analysis will be employed to regress for the effect of patient and tumour-related factors, surgical or medical complications on postoperative psychiatric morbidity. Key preoperative psychological factors associated with postoperative psychiatric morbidity will be identified by regression analysis.

\section{Dissemination}

Different methods of dissemination will be employed so that the findings from this research will reach patients, primary care practitioners, scientists, hospital specialists in gastroenterology, oncology and surgery, health policymakers and commissioners as well as healthcare regulatory bodies. The study findings will be presented at international gastroenterology, oncology and surgical research meetings. The findings of this research will also be presented to relevant patient groups. Ultimately, we plan to publish the results of this research in a high impact clinical journal to allow widespread dissemination of this research.

\section{Author affiliations}

${ }^{1}$ Department of Surgery \& Cancer, Imperial College London, London, UK

${ }^{2}$ London Psychometric Laboratory, University College London, London, UK

${ }^{3}$ Esophageal and Digestive Tract Surgical Unit, Veneto Institute of Oncology,

Padova, Veneto, Italy

${ }^{4}$ Department of Psychology, University of Lille, Lille, France

${ }^{5}$ Department of Digestive and Oncology surgery, University Hospital Claude

Huriez, Lille, France

${ }^{6}$ Department of Molecular Medicine \& Surgery, Karolinska Institutet, Karolinska University Hospital, Stockholm, Sweden

Contributors SRM was involved in study design and drafting of full protocol. SM took part in study conception, design of psychological constructs, sample size calculation and drafting of full protocol. KVP took part in study design and revising full protocol. MS, CC and CM were involved in study design and revising full protocol. VC was involved in study design, evaluation of psychological constructs and revising full protocol. PL and GBH took part in study conception, design and revising full protocol. GBH also took part in drafting of full protocol.

Funding SRM is supported by the National Institute for Health Research (NIHR); grant number (NIHR-CTF-2015-04-09).

Competing interests None declared.

Ethics approval NHS Health Research Authority (NRES committee LondonCentral Research Ethics Committee) approval gained 26 January 2016 (REC reference 15.LO/1847).

Provenance and peer review Not commissioned; externally peer reviewed.

Open Access This is an Open Access article distributed in accordance with the Creative Commons Attribution Non Commercial (CC BY-NC 4.0) license, which permits others to distribute, remix, adapt, build upon this work noncommercially, and license their derivative works on different terms, provided the original work is properly cited and the use is non-commercial. See: http:// creativecommons.org/licenses/by-nc/4.0/

\section{REFERENCES}

1. Bouras G, Markar SR, Burns E, et al. Linked hospital and primary care database analysis of the incidence and impact of psychiatric morbidity following gastrointestinal cancer surgery in England. Ann Surg 2016;264:93-9.

2. Voigt $\mathrm{V}$, Neufeld $\mathrm{F}$, Kaste $\mathrm{J}$, et al. Clinically assessed posttraumatic stress in patients with breast cancer during the first year after diagnosis in the prospective, longitudinal, controlled COGNICARES study. Psychooncology 2016. Published Online First: 22 Feb 2016. doi: 10.1002/pon.4102

3. Granot M, Ferber SG. The roles of pain catastrophizing and anxiety in the prediction of postoperative pain intensity: a prospective study. Clin J Pain 2005;21:439-45.

4. Powell R, Scott NW, Manyande A, et al. Psychological preparation and postoperative outcomes for adults undergoing surgery under general anaesthesia. Cochrane Database Syst Rev 2016;5:CD008646.

5. Lehto US, Ojanen M, Dyba T, et al. Baseline psychosocial predictors of survival in localized breast cancer. Br J Cancer 2006;94:1245-52.

6. Montazeri A, Gillis CR, McEwen J. Quality of life in lung cancer patients. A review of literature from 1970-1995. Chest 1998;113:467-81.

7. Walker MS, Zona DM, Fisher EB. Depressive symptoms after lung cancer surgery: their relation to coping style and social support. Psychooncology 2006;15:684-93. 
8. Chida $\mathrm{Y}$, Hamer M, Wardle J, et al. Do stress-related psychological factors contribute to cancer incidence and survival? Nat Clin Pract Oncol 2008;5:466-75.

9. Nakaya N, Bidstrup PE, Saito-Nakaya K, et al. Personality traits and cancer risk and survival based on Finnish and Swedish registry data. Am J Epidemiol 2010;172:377-85.

10. Adelita V, Ranchor AV, Sanderman R, Coyne J. Invited commentary: personality as a causal factor in cancer risk and mortality - time to retire a hypothesis? Am J Epidemiol 2010;172:386-8.

11. Satin JR, Linden W, Phillips MJ. Depression as a predictor of disease progression and mortality in cancer patients: a meta-analysis. Cancer 2009;111:5349-61.

12. Nakaya N. Effect of psychosocial factors on cancer risk and survival $J$ Epidemiol 2014;24:1-6.

13. Wikman A, Ljung R, Johar A, et al. Psychiatric morbidity and survival for esophageal cancer: a population-based cohort study. J Clin Oncol 2015;33:448-54.

14. Falaga ME, Zarkadoulia EA, loannidou EN, et al. The effect of psychosocial factors on breast cancer outcome: a systematic review. Breast Cancer Res 2007;9:R44.

15. Petticrew M, Bell R, Hunter D. Influence of psychological coping on survival and recurrence in people with cancer: a systematic review. BMJ 2002;325:1066

16. Garssen B. Psychological factors and cancer development: evidence after 30 years of research. Clin Psychol Rev 2004;24:315-38.

17. Nausheen B, Gidron Y, Peveler R, et al. Social support and cancer progression: a systematic review. J Psychosom Res 2009;67:403-15.

18. Smith S, Petrides KV, Green J, et al. The role of trait emotional intelligence in the diagnostic cancer pathway. Support Care Cancer 2012;20:2933-9.

19. Smith S, Turner B, Pati J, et al. Psychological impairments in patients urgently referred for prostate and bladder cancer evaluations: the role of trait emotional intelligence and perceived social support. Support Care Cancer 2012;20:699-704.

20. Antoni $\mathrm{MH}$. Psychosocial intervention effects on adaptation, disease course and biobehavioral processes in cancer. Brain Behav Immun 2013;30:S88-98

21. Greer S, Moorey S, Baruch JD, et al. Adjuvant psychological therapy for patients with cancer: a prospective randomized trial. BMJ 1992;304:675-80.
22. Johnston M, Vögele C. Benefits of psychological preparation for surgery: a meta-analysis. Ann Behav Med 1993;15:245-56.

23. Moorey S, Greer S, Watson M, et al. Adjuvant psychological therapy for patients with cancer: outcome at one year. Psychooncology 1994;3:39-46.

24. Artinyan A, Orcutt ST, Anaya DA, et al. Infectious postoperative complications decrease long-term survival in patients undergoing curative surgery for colorectal cancer: a study of 12,075 patients. Ann Surg 2015;261:497-505.

25. Derogar M, Orsini N, Sadr-Azodi O, et al. Influence of major postoperative complications on health-related quality of life among long-term survivors of esophageal cancer surgery. J Clin Oncol 2012;30:413-18.

26. Petrides KV. Technical manual for the Trait Emotional Intelligence Questionnaires (TEIQue). London: London Psychometric Laboratory, 2009.

27. Beck AT, Steer RA, Brown GK. Manual for the Beck Depression Inventory-II. San Antonio, TX: Psychological Corporation, 1996.

28. Beck AT, Ward $\mathrm{CH}$, Mendelson $\mathrm{M}$, et al. An inventory for measuring depression. Arch Gen Psychiatry 1961;4:561-71.

29. Carver CS. COPE Inventory. Measurement Instrument Database for the Social Science. 2013.

30. Spielberger CD. State-Trait Anxiety Inventory: bibliography. 2nd ed. Palo Alto, CA: Consulting Psychologists Press, 1989.

31. Boyle GJ, Saklofske DH, Matthews G. eds. Measures of personality and social psychological constructs. Elsevier/Academic Press, 2015.

32. Petrides KV. An application of belief-importance theory with reference to trait emotional intelligence, mood, and somatic complaints. Scand J Psychol 2011;52:161-7.

33. Low DE, Alderson D, Cecconello I, et al. International consensus on standardization of data collection for complications associated with esophagectomy: esophagectomy complications consensus group (ECCG). Ann Surg 2015;262:286-94.

34. Whistance RN, Conroy $\mathrm{T}$, Chie $\mathrm{W}$, et al. Clinical and psychometric validation of the EORTC QLQ-CR29 questionnaire module to assess health-related quality of life in patients with colorectal cancer. Eur J Cancer 2009;45:3017-26.

35. Blazeby JM, Farndon JR, Donovan J, et al. A prospective longitudinal study examining the quality of life of patients with esophageal carcinoma. Cancer 2000;88:1781-7.

36. Blazeby JM, Conroy T, Bottomley A, et al. Clinical and psychometric validation of a questionnaire module, the EORTC QLQ-STO 22, to assess quality of life in patients with gastric cancer. Eur $J$ Cancer 2004;40:2260-8. 